\title{
Comparison of ABC Methods with Computerized Estimates of Intracerebral Hemorrhage Volume: The INTERACT2 Study
}

\author{
Candice Delcourt ${ }^{a-c}$ Cheryl Carcel ${ }^{a, b}$ Danni Zheng ${ }^{a, b}$ Shoichiro Sato ${ }^{a, d}$ \\ Hisatomi Arima ${ }^{a}$ e Sonu Bhaskar ${ }^{b, f}, g$ Pierre Janin ${ }^{b, h}$ Rustam Al-Shahi Salman ${ }^{i}$ \\ Yongjun Cao ${ }^{j}$ Shihong Zhang ${ }^{k}$ Emma Heeleyl Leo Davies ${ }^{b, c}$ John Chalmers ${ }^{a}$ \\ Craig S. Anderson ${ }^{a, c}$ m INTERACT2 Investigators \\ aThe George Institute for Global Health, Faculty of Medicine, UNSW, Sydney, NSW, \\ Australia; ${ }^{b}$ Sydney Medical School, University of Sydney, Sydney, NSW, Australia; 'Neurology \\ Department, Royal Prince Alfred Hospital, Sydney Health Partners, Sydney, NSW, Australia;

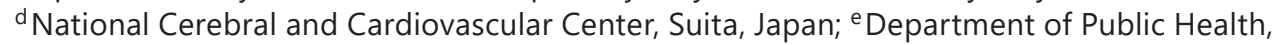 \\ Fukuoka University, Fukuoka, Japan; ${ }^{\mathrm{f} N e u r o l o g y ~ D e p a r t m e n t, ~ L i v e r p o o l ~ H o s p i t a l, ~ S y d n e y, ~}$ \\ NSW, Australia; 9 Ingham Institute for Applied Medical Research, UNSW, Sydney, NSW,

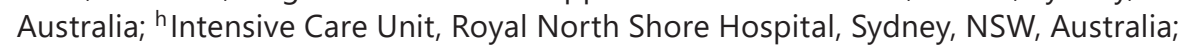 \\ 'Centre for Clinical Brain Sciences, University of Edinburgh, Edinburgh, UK; ${ }^{j}$ Department of \\ Neurology, Second Affiliated Hospital of Soochow University, Suzhou, China; ${ }^{\text {NNeurological }}$ \\ Department, West China Hospital, Sichuan University, Chengdu, China; 'Data Intelligence, \\ Strategic Research Investment, Cancer Institute NSW, Sydney, NSW, Australia; ${ }^{\mathrm{m}}$ The George \\ Institute China, Peking University Health Sciences Center, Beijing, China
}

\section{Keywords}

Intracerebral hemorrhage $\cdot$ Hematoma size $\cdot$ Imaging, stroke $\cdot$ Stroke outcome measures

\section{Abstract}

Background and Purpose: Hematoma volume is a key determinant of outcome in acute intracerebral hemorrhage $(\mathrm{ICH})$. We aimed to compare estimates of $\mathrm{ICH}$ volume between simple $(\mathrm{ABC} / 2$, length, width, and height) and gold standard planimetric software approaches. Methods: Data are from the second Intensive Blood Pressure Reduction in Acute Cerebral Hemorrhage Trial (INTERACT2). Multivariable linear regression was used to compare ICH volumes on baseline $C T$ scans using the $A B C / 2$, modified $A B C / 2(m A B C / 2)$, and MIStar software. Other aspects of $\mathrm{ICH}$ morphology examined included location, irregularity, heterogeneity,

Clinical Trial Registration: INTERACT2 is registered at ClinicalTrials.gov (NCT00716079). 
intraventricular and subarachnoid hemorrhage extension (SAH) of hematoma, and associated white matter lesions and brain atrophy. Results: In 2,084 patients with manual and semiautomated measurements, median (IQR) ICH volumes for each approach were: ABC/2 11.1 (5.11$20.88 \mathrm{~mL}), \mathrm{mABC} / 27.8$ (3.88-14.11 mL), and MIStar 10.7 (5.59-18.66 mL). Median differences between $A B C / 2$ and MIStar, and $\mathrm{mABC} / 2$ and MIStar were 0.34 (-1.01 to 2.96) and $-2.4(-4.95$ to -0.7416), respectively. Hematoma volumes differed significantly with irregular shape $(A B C / 2$ and MIStar, $p<0.001 ; \mathrm{mABC} / 2$ and MIStar, $p=0.007)$ and larger volumes $(\mathrm{mABC} / 2$ and MIStar, $p<0.001 ; A B C / 2$ and MIStar, $p=0.07)$. ICH with SAH showed a significant discrepancy between $A B C / 2$ and MIStar ( $p<0.001$ ). Conclusions: Overall, ABC/2 performs better than $\mathrm{mABC} / 2$ in estimating $\mathrm{ICH}$ volume. The largest discrepancies were evidenced against automated software for irregular-shaped and large $\mathrm{ICH}$ with $\mathrm{SAH}$, but the clinical significance of this is uncertain.

\section{Introduction}

Intracerebral hemorrhage ( $\mathrm{ICH})$ is the most severe type of stroke with case fatality ranging from 30 to 50\% [1]. Age, clinical severity, and brain imaging features influence outcomes. Hematoma volume is the strongest predictor of 30-day mortality and a strong prognostic factor for patients with acute ICH [2]. Whilst various computer-assisted semiautomated methods using Hounsfield unit threshold segmentation [3, 4] for determining hematoma volume are gaining popularity in research and clinical settings, the simple $\mathrm{ABC} / 2$ method, which combines the largest measure in each of 3 dimensions of brain imaging slices, is most often used to derive a formal estimate of hematoma volume in this critical illness. The method was first reported by Kwak et al. [5] and Broderick et al. [6], and later validated by Kothari et al. [7], who suggested a variation to derive the $C$ value for increased accuracy, the modified $\mathrm{ABC} / 2(\mathrm{mABC} / 2)$. However, as the $\mathrm{ABC} / 2$ method is based on the assumption that ICH has a bipyramidal ellipsoid shape, it may under- or overestimate actual ICH volume [8, 9], particularly in an irregular shape [9-11]. Herein, we aimed to quantify differences between the $2 \mathrm{ABC} / 2$ methods and semiautomated multislice techniques using MIStar ${ }^{\mathrm{TM}}$ (Apollo Inc, Melbourne, VIC, Australia) software for participants of the second Intensive Blood Pressure Reduction in Acute Cerebral Hemorrhage Trial (INTERACT2; the list of all study investigators is listed in the supplementary material; for all online supplementary material, see www. karger.com/doi/10.1159/000504531) [12].

\section{Methods}

\section{Design}

INTERACT2 was an international, multicenter, open, blinded endpoint, randomized controlled trial, as described in detail elsewhere [12,13]. In brief, 2,839 patients with spontaneous ICH within $6 \mathrm{~h}$ of onset and elevated systolic blood pressure (SBP, 150-220 $\mathrm{mm} \mathrm{Hg}$ ) were randomly assigned to receive intensive (target SBP $<140 \mathrm{~mm} \mathrm{Hg}$ within $1 \mathrm{~h}$ ) or guideline-recommended (target SBP $<180 \mathrm{~mm} \mathrm{Hg}$ ) blood pressure-lowering treatment. Demographic and clinical characteristics were recorded at the time of enrolment (baseline) of patients, with their neurological severity measured with the Glasgow Coma Scale and the National Institutes of Health Stroke Scale (NIHSS). The study protocols were approved by appropriate ethics committees at each site, and written informed consent was obtained from each patient or where appropriate, an approved surrogate. INTERACT2 is registered at ClinicalTrials.gov (NCT00716079). 


\section{Procedures for Measuring ICH Parameters}

Baseline computed tomography (CT) scans were obtained according to standardized techniques and analyzed centrally by blinded assessors, where hematoma volume was determined using computer-assisted multislice morphometric and voxel threshold techniques using MIStar version 3.2 (Apollo Medical Imaging Inc, Melbourne, VIC, Australia). Briefly, on 1 axial slice, a region of interest was drawn within the ICH manually by an assessor: the Hounsfield unit range selected to include density of blood and region of interest "grown" automatically to enclose the ICH on each slice. The software automatically applied thickness to calculate volume on each slice with volumes added up manually. Intraventricular blood was not included. Calcified structures included with a density close to blood were manually subtracted. Times invested in analyzing each CT scan varied from $30 \mathrm{~s}$ to $3 \mathrm{~min}$ according to the complexity of ICH and its proximity to the cranium and calcified structures.

For this study, all CT scans had ICH volumes re-analyzed using both $\mathrm{ABC} / 2$ methods by 3 independent neurologists (online suppl. Figure S1). Briefly, brain CT scan slices were first screened to measure the largest diameter A of hematoma, which was followed by measuring the largest diameter perpendicular to A on the same slice to yield B. Finally, the number of slices on which hematoma was seen was tabulated and multiplied by slice thickness C. For the $\mathrm{mABC} / 2$ approach, $\mathrm{C}$ was modified by comparing each CT slice with hemorrhage to the CT slice with the largest hemorrhage; if this was $>75 \%$ on the slice with the largest volume, a weighting of 1 was given in determining C; if the volume was in the range of $25-75 \%$ of the maximum slice area, a weighting of 0.5 was given; and if the area was $<25 \%$ of the largest hemorrhage, the slice was excluded from analysis. These weighted values of CT containing hemorrhages were then summed to determine the value for $C$, which was multiplied by slice thickness [7]. Given these methods discard slices at the margins of the hematoma, they are expected to yield smaller true volumes. Measurements for A and B were made on a linear scale (in $\mathrm{cm}$ ) in MIStar, with A, B, and C then multiplied for the product to be divided by 2 , to yield an estimated hemorrhage volume in milliliters.

Irregularity of shape and heterogeneity of density (clot density variation) for ICH were assessed using the scale of Barras et al. [14], with scores assigned from 1 (most regular shape or most homogeneous density) to 5 (most irregular shape or most heterogeneous density). For analytical purposes, these measures were dichotomized as heterogeneous/irregular (scores 3-5) or homogeneous/regular (scores 1-2). Brain atrophy was measured using linear measurements and visual rating from templates, as described elsewhere [15]; the presence of any atrophy defined on at least one scale. The presence of any white matter lesion was defined as a total score $\geq 1$ on the scale by van Swieten et al. [16] from measures taken in the hemisphere contralateral to the ICH by location (anterior, posterior) and extent (absent [score $=0]$, partly involving the white matter [score $=1]$, or extending up to the subcortical region $[$ score $=2]$ ).

\section{Statistical Analyses}

Continuous baseline variables were compared using the Student $t$ test and Wilcoxon rank-sum test, and expressed as means \pm SD or medians (IQR). Categorical variables were compared using the $\chi^{2}$ test. ICH imaging parameters (lobar location, volume, irregular shape, heterogeneous density, intraventricular extension, subarachnoid extension, white matter lesions, and atrophy) contributing to differences between the planimetric measurement and $\mathrm{ABC} / 2$ were assessed in crude and multivariable linear regression models. Variables were included in the multivariable model if they were significant $(p<0.1)$ in the crude analyses. Discrepancies were defined as percentages (ABC/2-MIStar/MIStar and mABC/2-MIStar/ MIStar). Scatter plots were used to visualize the relationship between the discrepancy in volume measured by the $\mathrm{ABC} / 2$ and $\mathrm{mABC} / 2$ methods and MIStar measures and baseline 
Table 1. Crude and adjusted linear regression analyses for discrepancies between mABC/2 and MIStar methods (mABC/2 - MIStar) for estimating volume of intracerebral hemorrhage (ICH)

\begin{tabular}{|c|c|c|c|c|c|c|c|c|}
\hline \multirow[t]{2}{*}{ ICH features } & \multicolumn{4}{|l|}{ Crude } & \multicolumn{4}{|l|}{ Adjusted } \\
\hline & $\beta$ & $95 \% \mathrm{CI}$ & & $p$ value & $\beta$ & $95 \% \mathrm{CI}$ & & $p$ value \\
\hline Lobar location & -2.190 & -3.194 & -1.185 & $<0.001$ & 0.2419 & -0.7648 & 1.2485 & 0.6376 \\
\hline Volume, mL & -2.994 & -3.259 & -2.729 & $<0.001$ & -3.3039 & -3.6371 & -2.9707 & $<0.001$ \\
\hline Irregular shape & -0.575 & -1.176 & 0.027 & 0.061 & 1.8723 & 1.2903 & 2.4542 & $<0.001$ \\
\hline Heterogenous density & -1.654 & -2.268 & -1.040 & $<0.001$ & 0.1273 & -0.4607 & 0.7153 & 0.6712 \\
\hline Intraventricular extension & -0.751 & -1.413 & -0.090 & 0.026 & -0.0607 & -0.6788 & 0.5573 & 0.8472 \\
\hline Subarachnoid extension & -4.115 & -5.202 & -3.028 & $<0.001$ & -1.4648 & -2.5513 & -0.3782 & 0.0083 \\
\hline White matter lesion & 0.871 & 0.264 & 1.479 & 0.005 & 0.6500 & 0.0803 & 1.2197 & 0.0254 \\
\hline Atrophy & 0.434 & -0.355 & 1.223 & 0.281 & -0.4020 & -1.2034 & 0.3994 & 0.3254 \\
\hline
\end{tabular}

CI, confidence interval. Volume data were log transformed. Model adjusted for age, sex, ethnicity, high National Institutes of Health Stroke Scale score ( $<14$ vs. $\geq 14$ ), and history of ischemic stroke, hypertension, and antithrombotic use.

Table 2. Crude and adjusted linear regression analyses for discrepancies between ABC/2 and MIStar (ABC/2-MIStar) for estimating volume of intracerebral hemorrhage (ICH)

\begin{tabular}{|c|c|c|c|c|c|c|c|c|}
\hline \multirow[t]{2}{*}{ ICH features } & \multicolumn{4}{|l|}{ Crude } & \multicolumn{4}{|l|}{ Adjusted } \\
\hline & $\beta$ & $95 \% \mathrm{CI}$ & & $p$ value & $\beta$ & $95 \% \mathrm{CI}$ & & $p$ value \\
\hline Lobar location & 4.181 & 2.730 & 5.632 & $<0.001$ & 0.8269 & -0.7504 & 2.4041 & 0.304 \\
\hline Volume, mL & 1.588 & 1.165 & 2.011 & $<0.001$ & 0.2888 & -0.2274 & 0.8050 & 0.273 \\
\hline Irregular shape & 3.787 & 2.931 & 4.644 & $<0.001$ & 2.5545 & 1.6466 & 3.4624 & $<0.001$ \\
\hline Heterogeneous density & 2.050 & 1.159 & 2.942 & $<0.001$ & 0.9493 & 0.0279 & 1.8708 & 0.044 \\
\hline Intraventricular extension & 1.162 & 0.203 & 2.120 & 0.018 & 0.3953 & -0.5675 & 1.3580 & 0.421 \\
\hline Subarachnoid extension & 7.268 & 5.703 & 8.833 & $<0.001$ & 5.1520 & 3.4574 & 6.8466 & $<0.001$ \\
\hline White matter lesion & 0.968 & 0.086 & 1.850 & 0.031 & 0.6005 & -0.2923 & 1.4933 & 0.187 \\
\hline Atrophy & -0.339 & -1.483 & 0.805 & 0.562 & -1.8004 & -3.0520 & -0.5488 & 0.005 \\
\hline
\end{tabular}

CI, confidence interval. Volume data were log transformed. Model adjusted for age, sex, ethnicity, high National Institutes of Health Stroke Scale score ( $<14$ vs. $\geq 14$ ), and history of ischemic stroke, hypertension, and antithrombotic use.

volume. Scatter plots were also used to visualize the relationship between the volume discrepancies and ICH shape measured by the Barras scale. All data were analyzed using SAS software (version 9.3; SAS Institute, Cary, NC, USA).

\section{Results}

Among the 2,829 INTERACT2 participants, 2,084 (74\%) had available brain CT scans in a format suitable for hematoma volume measurement using semiautomated (MIStar) and manual $(\mathrm{ABC} / 2$ and $\mathrm{mABC} / 2)$ methods. CT scans of 745 patients were excluded from $\mathrm{ABC}$ measurements because the examiners judged them to be too complex and most $(740 ; 99 \%)$ had an infratentorial ICH location. Included patients were less often of Chinese ethnicity (66.4 vs. $71.9 \% ; p=0.006$ ), were randomized earlier ( 3.7 vs. $3.8 \mathrm{~h} ; p=0.009$ ), and had higher median Glasgow Coma Scale scores (14 [13-15) vs. 14 [12-15); $p=0.001$ ), smaller hematomas on planimetric measurements ( 10.7 vs. $12 \mathrm{~mL} ; p<0.001$ ) and SAH extension ( 8 vs. $5 \%$, $p=0.01$ ) than those excluded (online suppl. Table S1). 


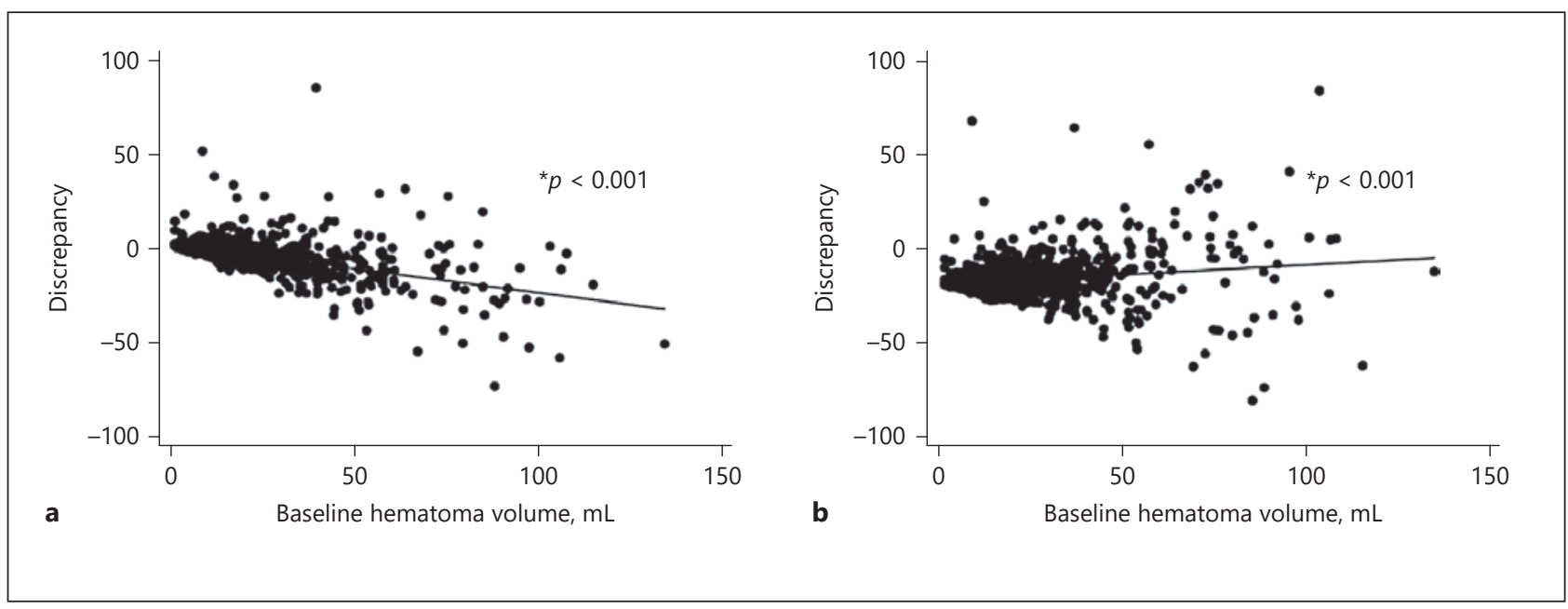

Fig. 1. Association between baseline hematoma volume and measurement discrepancy. Linear regression model. a ABC/2 versus MIStar. Discrepancy $=0.28692-0.25488 \times$ hematoma volume $\left(r^{2}=0.3131\right)$. A positive correlation for differences between the ABC/2 method and MIStar and increasing volume of ICH (measured using MIStar) is seen with larger discrepancies when ICH volume increases. $\mathbf{b} \mathrm{mABC} / 2$ versus MIStar. Discrepancy $=-0.18106+0.13183 \times$ hematoma volume $\left(r^{2}=0.0399\right)$. A positive correlation for differences between the $\mathrm{mABC} / 2$ method and MIStar and increasing volume of ICH with larger discrepancies is seen when ICH volume increases.
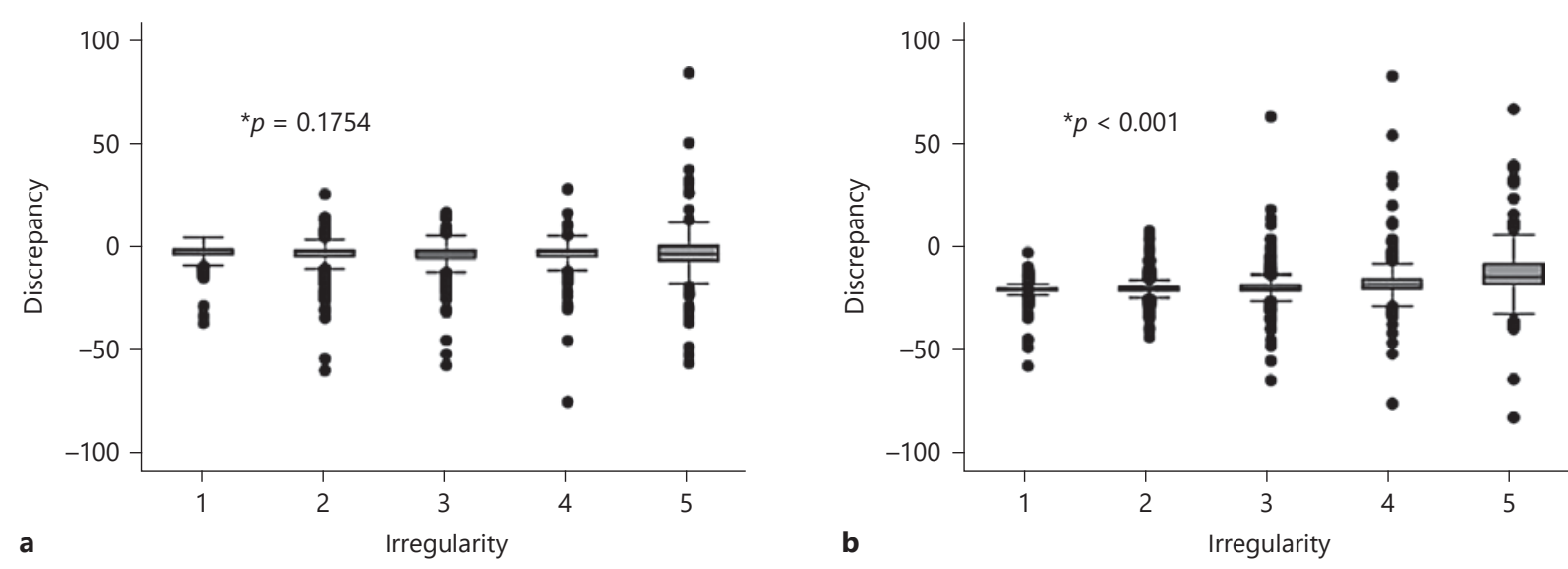

Fig. 2. Association between baseline hematoma irregularity and measurement discrepancy. Linear regression model. a ABC/2 versus MIStar. Discrepancy $=-3.10078-0.17191 \times$ shape score $\left(r^{2}=0.0009\right)$. The largest differences between $\mathrm{ABC} / 2$ and MIStar are seen with irregular ICH, corresponding to Barras scale scores $3-5$. b $\mathrm{mABC} / 2$ versus MIStar. Discrepancy $=-3.92960+2.24211 \times$ shape score $\left(r^{2}=0.0714\right)$. The largest differences between the $\mathrm{mABC} / 2$ and MIStar are seen with irregular ICH, corresponding to Barras scale score 3-5.

The median volumes across $\mathrm{ABC} / 2, \mathrm{mABC} / 2$, and MIStar measures were 11.1 (5.1120.88), 7.8 (3.88-14.11), and $10.7 \mathrm{~mL}$ (5.59-18.66), respectively. Median differences between $\mathrm{ABC} / 2$ and MIStar (ABC/2-MIStar) were $0.34 \mathrm{~mL}(-1.01$ to 2.96$)$ and between $\mathrm{mABC} / 2$ and MIStar (mABC/2-MIStar) $-2.4 \mathrm{~mL}(-4.95$ to -0.7416$)$ (online suppl. Tables $\mathrm{S} 2, \mathrm{~S} 3)$. 
Tables 1 and 2 provide results of crude and multivariable linear regression analyses. ICH cases showing the biggest differences in volumes between both methods were those that were irregular and associated with SAH extension. Figure 1 shows a positive correlation for differences between both methods and increasing ICH volume with larger discrepancies when ICH volume increases. Figure 2 illustrates the differences by shape according to the Barras scoring system. Irregular ICH, corresponding to Barras scale score $3-5$, showed the largest differences between the 2 methods.

\section{Discussion}

These secondary analyses of the INTERACT2 imaging database show that estimated volumes of acute ICH in cases where the hematoma is round to ellipsoid in shape without SAH extension are comparable utilizing the $\mathrm{ABC} / 2$ and $\mathrm{mABC} / 2$ approaches against a semiautomated measurement. However, significant differences emerge when the hematoma is large and irregular, with $\mathrm{mABC} / 2$ tending to underestimate volume due to its modified method of deriving the $C$ value. However, any measurement error of volume is small $(1-2 \mathrm{~mL})$ and unlikely to be clinically important for most ICH cases, as such a difference is estimated to translate into $5-10 \%$ increased odds of poor outcome $[14,15]$.

The difference seen between conventional $\mathrm{ABC} / 2$ and MIStar for regular ICH is similar to a report by Kothari et al. [7] in 118 cases, where the simple method performs well for most ICH types. There are many reports of differences in the approaches for patients with large and irregular ICH $[6-8,16]$, and this appears greater for hematomas in a lobar location $[4,7,16]$. In INTERACT2, lobar ICH was present in only $10 \%$ of cases and did not influence measurement error in our analyses. This was not the case for SAH expansion, which produces technical challenges in delimitating from ICH when it is often irregular. Although white matter lesions have been shown to be associated with large ICH, possibly from rarefaction of brain tissue and blood brain barrier damage [17], the only differences in hematoma volumes introduced were between the $\mathrm{mABC} / 2$ and planimetric methods.

Strengths of our analysis include the large dataset derived from a pragmatic international multicenter study with systematic data collection and rigorous central analysis of imaging. However, we had to exclude cases of infratentorial ICH from analyses due an inability to clearly define the hematoma border, introducing further selection bias in a clinical trial population of hypertensive, predominantly mild/moderately severe ICH. Finally, these were post hoc analyses subject to chance associations and incomplete adjustment for confounders.

In summary, the easy-to-use $\mathrm{ABC} / 2$ method provides an accurate estimate of hematoma volume in mild/moderately sized, ellipsoid-shaped ICH. While measurement error is apparent for large and irregular ICH, an accurate measurement of hematoma volume may be less relevant in such cases due to the overall poor prognosis [1]. The alternative, more complicated $\mathrm{mABC} / 2$ method consistently underestimates hematoma volumes and is not recommended for practice or research.

\section{Statement of Ethics}

The study protocols were approved by appropriate ethics committees at each site, and written informed consent was obtained from each patient or where appropriate, an approved surrogate. 


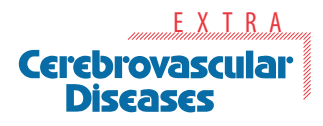

\begin{tabular}{l|l}
\hline Cerebrovasc Dis Extra 2019;9:148-154 \\
\hline DOI: 10.1159/000504531 & $\begin{array}{l}\text { @ 2019 The Author(s). Published by S. Karger AG, Basel } \\
\text { www.karger.com/cee }\end{array}$ \\
\hline
\end{tabular}

Delcourt et al.: The INTERACT2 Study

\section{Disclosure Statement}

C.D. holds a National Heart Foundation of Australia Postdoctoral Fellowship. S.S. held a fellowship from the Japan Brain Foundation during these analyses and is an employee of Bayer, Japan. J.C. has received research grants from NHMRC and Idorsia. C.S.A. holds a Senior Principal Research Fellowship of the NHMRC of Australia, and reports receiving travel reimbursement and honorarium from Takeda China, Boehringer Ingelheim, and Amgen. C.C., D.Z, H.A., S.B., P.J., R.A.-S.S., Y.C., S.Z., E.H., and L.D. report no disclosures.

\section{References}

1 Feigin VL, Lawes CM, Bennett DA, Barker-Collo SL, Parag V. Worldwide stroke incidence and early case fatality reported in 56 population-based studies: A systematic review. Lancet Neurol. 2009 Apr;8(4):355-69.

2 Broderick JP, Brott TG, Duldner JE, Tomsick T, Huster G. Volume of intracerebral hemorrhage. A powerful and easy-to-use predictor of 30-day mortality. Stroke. 1993 Jul;24(7):987-93.

3 Kosior JC, Idris S, Dowlatshahi D, Alzawahmah M, Eesa M, Sharma P, et al.; PREDICT/Sunnybrook CTA ICH Study Investigators. Quantomo: Validation of a computer-assisted methodology for the volumetric analysis of intracerebral haemorrhage. Int J Stroke. 2011 Aug;6(4):302-5.

4 Wu TY, Sobowale O, Hurford R, Sharma G, Christensen S, Yassi N, et al. Software output from semi-automated planimetry can underestimate intracerebral haemorrhage and peri-haematomal oedema volumes by up to 41 . Neuroradiology. 2016 Sep;58(9):867-76.

5 Kwak R, Kadoya S, Suzuki T. Factors affecting the prognosis in thalamic hemorrhage. Stroke. 1983 Jul-Aug; 14(4):493-500.

6 Broderick JP, Brott TG, Tomsick T, Barsan W, Spilker J. Ultra-early evaluation of intracerebral hemorrhage. J Neurosurg. 1990 Feb;72(2):195-9.

7 Kothari RU, Brott T, Broderick JP, Barsan WG, Sauerbeck LR, Zuccarello M, et al. The ABCs of measuring intracerebral hemorrhage volumes. Stroke. 1996 Aug;27(8):1304-5.

8 Sheth KN, Cushing TA, Wendell L, Lev MH, Romero JM, Schwab K, et al. Comparison of hematoma shape and volume estimates in warfarin versus non-warfarin-related intracerebral hemorrhage. Neurocrit Care. 2010 Feb;12(1):30-4.

9 Freeman WD, Barrett KM, Bestic JM, Meschia JF, Broderick DF, Brott TG. Computer-assisted volumetric analysis compared with $\mathrm{ABC} / 2$ method for assessing warfarin-related intracranial hemorrhage volumes. Neurocrit Care. 2008;9(3):307-12.

10 Huttner HB, Steiner T, Hartmann M, Köhrmann M, Juettler E, Mueller S, et al. Comparison of ABC/2 estimation technique to computer-assisted planimetric analysis in warfarin-related intracerebral parenchymal hemorrhage. Stroke. 2006 Feb;37(2):404-8.

11 Krishnan K, Mukhtar SF, Lingard J, Houlton A, Walker E, Jones T, et al. Performance characteristics of methods for quantifying spontaneous intracerebral haemorrhage: Data from the Efficacy of Nitric Oxide in Stroke (ENOS) trial. J Neurol Neurosurg Psychiatry. 2015 Nov;86(11):1258-66.

12 Anderson CS, Heeley E, Huang Y, Wang J, Stapf C, Delcourt C, et al.; INTERACT2 Investigators. Rapid bloodpressure lowering in patients with acute intracerebral hemorrhage. N Engl J Med. 2013 Jun;368(25):2355-65.

13 Delcourt C, Huang Y, Wang J, Heeley E, Lindley R, Stapf C, et al.; INTERACT2 Investigators. The second (main) phase of an open, randomised, multicentre study to investigate the effectiveness of an intensive blood pressure reduction in acute cerebral haemorrhage trial (INTERACT2). Int J Stroke. 2010 Apr;5(2):110-6.

14 Barras CD, Tress BM, Christensen S, MacGregor L, Collins M, Desmond PM, et al.; Recombinant Activated Factor VII Intracerebral Hemorrhage Trial Investigators. Density and shape as CT predictors of intracerebral hemorrhage growth. Stroke. 2009 Apr;40(4):1325-31.

15 Sato S, Delcourt C, Heeley E, Arima H, Zhang S, Al-Shahi Salman R, et al.; INTERACT2 Investigators. Significance of cerebral small-vessel disease in acute intracerebral hemorrhage. Stroke. 2016 Mar;47(3):701-7.

16 van Swieten JC, Hijdra A, Koudstaal PJ, van Gijn J. Grading white matter lesions on CT and MRI: A simple scale. J Neurol Neurosurg Psychiatry. 1990 Dec;53(12):1080-3.

17 Lou M, Al-Hazzani A, Goddeau RP Jr, Novak V, Selim M. Relationship between white-matter hyperintensities and hematoma volume and growth in patients with intracerebral hemorrhage. Stroke. 2010 Jan;41(1):34-40. 